\title{
It Is Not What It Looks Like-A Response to D. J. Pons, A. D. Pons, and A. J. Pons Theory of Time
}

\author{
Kamil Łacina ${ }^{1}$ \\ ${ }^{1}$ Department of Philosophy, Jagiellonian University, Krakow, Poland \\ Correspondence: Kamil Łacina, Department of Philosophy, Jagiellonian University, Grodzka 52 Street, Krakow \\ 31-044, Poland. E-mail: kamillacina@yahoo.pl
}

Received: November 27, 2013

Accepted: December 13, 2013 Online Published: January 13, 2014

doi:10.5539/apr.v6n1p26

URL: http://dx.doi.org/10.5539/apr.v6n1p26

\begin{abstract}
The most recent theory of time introduced by D. J. Pons, A. D. Pons, and A. J. Pons in their paper "Time: An Emergent Property of Matter" states that time is an emergent property of matter. The theory, based on the non-local hidden-variable approach to quantum mechanics presents an account of time as a layered emergent property of particule frequency oscillation. The paper goes on to explain how time and the arrow of time emerge at different level of matter assembly, most notably in decoherent systems. The theory also addresses the issue of human time perception and, partly, the ontological issue of space-time substantivalism. The theory however seems to be suffering from some pitfalls, which I intend to delineate and examine. Although in the end the theory appears not to hold, it nevertheless offers an important new direction in the analysis of time, one that seems worth pursuing further. The following paper presents the original theory first, before moving on to consider objections.
\end{abstract}

Keywords: time, Cordus conjecture, cognition, petitio principi, critique, clocks, present, frequency

\section{Introduction}

In their most recent paper D. J. Pons, A. D. Pons, and A. J. Pons (2013b) present a new theory of time- time as an emergent property of matter. They approach the subject form a quantum mechanics (QM) perspective, more specifically the non-local hidden-variable interpretation (NLHV). The NLHV interpretation was created as an alternative to the Copenhagen interpretation, the Einstein-Podolski-Rosen critique, and Bell's theorem (Leggett, 2003).

It is within the bounds of the NLHV interpretation that one finds the Cordus conjecture-a theory that D. J. Pons, A. D. Pons, and A. J. Pons have been popularising approximately since 2011. It is on the basis of this conjecture that the new theory of time (among others, Note 1) has been developed. Before moving on to the theory of matter-emergent time, one must familiarise oneself with the Cordus conjecture itself.

The Cordus conjecture (according to D. J. Pons, A. D. Pons, \& A. J. Pons, 2013b) states that "all particles are one dimensional structures of finite length, and emit three-dimensional discrete forces at their two ends. This is called a particule. It is a NLHV design" (Pons et al., 2013b). This allows for a synchronous energising and de-energising of reactive ends of a particule, thus creating an oscillation frequency.

Based on these theoretic assumptions, authors present a new theory of matter-emergent time- a theory which will, in section 5, be proven untenable.

\section{Purpose of the Paper}

The following paper's main goal is not to blatantly criticise the work of D. J. Pons, A. D. Pons, and A. J. Pons. It is rather to improve the current body of knowledge regarding time by refuting an erroneous theory, thus putting us back on track of finding a sound and better one, through learning from mistakes of our predecessors.

\section{Method}

This paper makes use of two approaches, as it addresses areas that require certain academic versatility. First approach consists of a careful analysis of the solutions proposed by D. J. Pons, A. D. Pons, and A. J. Pons in light of academic literature (Block, 1980; Churchland, 2006; Dainton, 2011; Feigl, 1958; Gallagher, 2011; Lewin, 1935; Lyre, 2008; Nosal \& Bajcar, 2004; Nosal, 2006; Putnam, 1975). Second approach is a logical analysis of 
the entire theory based on its underlying assumption - and it is this analysis that ultimately leads me to reject the Cordus theory of time, on grounds that it falls prey to petitio principi fallacy.

\section{Analysis of Matter-Emergent Time Theory}

\subsection{Fundamental Time as Oscillation Frequency of a Particule}

In the matter-emergent time theory, time is divided into levels. As the size of a material entity increases, so emerge new levels of time. The most basic and most primal level of time (Level 1) is defined as the oscillation frequency of the reactive ends of a particule. This is so, because "accepting that time dilation does occur, and accepting also that atomic clocks do show a physical representation of that effect (as opposed to some other effect), then it is a reasonable assumption that at least the ticks of time are really represented in the mechanism of the clock" (Pons et al., 2013b). Therefore the energising and de-energising of reactive ends of a particule are interpreted as representing the "ticks" of time. The frequency of oscillation is said to be dependent on the particule - more specifically on its energy and mass. Particules with higher energy and/or mass oscillate faster than ones with lower energy/mass. (Pons et al., 2013b, p. 26) This is the most fundamental level of time, from which other levels will emerge. As the authors put it: "fundamental time, at the level of the individual particule (e.g. electron), is the frequency of the re-energisation cycles of its two reactive ends." (Pons et al., 2013b, p. 44)

\subsection{Coherent and Decoherent Assembly Level}

The next level features an important division: as the next level occurs at the particule-assembly into systems, it is crucial that we divide the second level of time into "coherent assembly level" and "decoherent assembly level"- a distinction on which the entire theory will be constructed.

Since a coherent system is composed of synchronised particles, the time such system represents is identical to the time represented by the oscillation frequency of a single particule. Moreover, a coherent system, much like a single particule, features no arrow of time - all of the processes are reversible. Therefore, a coherent system brings us no closer to the time that we experience on a daily basis. What we need is a decoherente system, which is quite convenient, as all of the complex material assemblages are in fact decoherente (Note 2).

A decoherent system presents us with a much more complex and intriguing picture. It is because of decoherence that time - as we know it — comes to be. When particules, of which a decoherent system is constructed, interact with each other, and, as a system, with the surrounding universe, a new level of time comes about. This new level (Level 2) is slower than the previous one and it has a unique direction-the arrow of time. This happens because as the particules interact with one another, they do so in an uncoordinated manner. This results in delays and mismatches in reactions of one particule energy transmission to the other. Moreover, as we are considering an uncoordinated, unsynchronised system, the distance matters. Small delays in interactions result from a finite speed (field propagation speed) at which information (forces) can be transferred across the manifold (or fabric). This transfer delay, coupled with the asynchronicity of particules of a decoherent system and the fact that the system interacts in-elastically with the universe (as well internally with oneself) renders any reversal either nearly infinitely improbable, or plainly impossible, as the number of variables that need to be reversed increases exponentially-hence the arrow of time (Note 3).

Before moving on to the third level of time, there are two important issues in need of mentioning: the fabric time and time-dilation.

"Fabric time is the mutual interconnectedness of matter particules spread over three-dimensional space. This occurs via the fabric, comprising discrete field forces for electric-magneto-gravitational interaction. Not strictly a time, this is rather a coordination of events across space." (Pons et al., 2013b, p. 32)

This fabric time offers no real ticks of time, representing rather the interconnectedness of particules of an accessible universe (each particule A receives discrete forces from particules in its past light cone). It also represents "a one-directional mutual causality" (Pons et al., 2013b). The prime importance of thus defined fabric is its relationship to the standard general relativity understanding of spacetime. It gives us a $3 \mathrm{D}$ space manifold with time defined as relationships between bodies. D. J. Pons, A. D. Pons, and A. J. Pons also point out that this theory of fabric, as carrying the electric-magnetic-gravitational (EMG) fields of particules makes spacetime quasi-substantial.

Time dilation in the Cordus theory is explained as retardation of a particule's frequency by the external environment. The more dens the fabric - external discrete forces - the slower the frequency oscillation of a particule. (Note 4) 


\subsection{Organic Life Level}

From here D. J. Pons, A. D. Pons, and A. J. Pons move on to the next level (Level 3) of time- the organic life-time. Once the way time emerges from a decoherent assembly of particules is established, we can examine how such systems join together to form an organism. Since a living organism is composed of cells and tissues - themselves decoherent assemblages - the previously considered assemblages of particules become sub-systems of a much more complex organic system. Following Cordus theory, and the fact that cells do not interact superluminally, we can see how the next level of time emerges, one that is much slower than the decoherent assembly time, by virtue of relying on chemical reactions speed.

"Organic-Life Time, at the level of an individual cell, consists of the nebulous aggregation of the discrete fields of the many individual coherent particules (electrons, atoms, molecules) and decoherent sub-components (clumps of molecules, organelles). 'Nebulous' because the discrete forces are not individually distinct. Chemical transport within the cell occurs as and when the sub-components are able to interact. Thus the cell takes much longer to achieve anything (more frequency ticks of its atoms) than a simple sum of the times required by the coherent subcomponents." (Pons et al., 2013b, p. 33)

This level of time is based in chemistry, as neurotransmitter molecules require much greater time to interact with other cells than particules require for interacting with one another. This chemical process dependence is characteristic of every living organism. Even the simplest of living organisms rely on chemical processes to exist. This chemical reaction time, specifically regarding neurotransmitter molecules, brings us to the next and final level of time - the cognitive time.

\subsection{Cognitive Level}

The final level (Level 4) of time is cognitive time, as experienced by human beings. According to the theory presented here, "human perception of time is therefore a cognitive construct that we overlay on chemical time and that in turn on the frequency of matter." (Pons et al., 2013b, p. 33) Authors refer us to an example of proprioception, which shows a cause-and-effect sequence, running one-way on chemical reactions/time. There is also a claim that such "cognitive construct", as is the human perception of time, is the cause for time's perceived universality. What is stated is that, at least at the level of action (praxis), time is taken to be universal by virtue of actions of different agents happening "at the same time" or in a temporally synchronised manner. To sum up:

"Cognitive time, or the human perception of time, is a cognitive construct of the order in which we experience phenomena, based in turn on a neural representation of chemical time, and the arrow of time (irreversibility), both of which arise at a deeper level. The coordination provided by the fabric ensures that there is a consistency of experience between two people, hence personal interaction is a real common experience, and time is perceived as universal.” (Pons et al., 2013b, p. 34)

What follows are some philosophical considerations which may be summed up as follows:

According to the matter-emergent time theory there is a single past and a single present which evolves into a single future. The present moment, or NOW, is "a cognitive effect associated with consciousness, memory, and the process of thought" and, in the same time it is "a summary of the many mutual interactions between particules throughout the universe" (Pons et al., 2013b). This leads to the statement that "there need only be one NOW that we all share and influence" (Pons et al., 2013b). Nevertheless, this theory does not reject the possible worlds theory (Note 5) outright, it rather states that such multiplicity of worlds is unnecessary.

The question of coordination of events, in the absence of some master clock, is answered by postulating that the fabric itself serves as a coordination device. The transmission of discrete forces happens within the fabric, thus allowing for a coordinated cause-and-effect structure of the universe.

"It is not a master clock that accomplishes the temporal connectedness of phenomena that are at different geometric locations, nor does it require continuity of spacetime per se. The piece-wise communication, via discrete field interactions of the fabric, between adjacent volumes of space (matter and fabric) applies spatial consistency to time." (Pons et al., 2013b, p. 35)

The picture of time painted by the Cordus theory is indeed an interesting and inspirational one. The multi-level approach to time proves not only feasible, but shows much promise in terms of agreeing such varied perspectives like quantum mechanics, general relativity, cognitive psychology, philosophy and biology. Unfortunately, there seem to be serious issues with this approach, some perhaps not anticipated by the authors. In the following section I will delineate what, in my opinion, disqualifies this theory of time. I will present three arguments: the first one relates to cognitive time, the second, to connectedness and fabric time, and the third, to the fundamental level of time, defined as oscillation frequency of a particule. 


\section{Analysis and Results}

Although initially it is tempting to offer arguments based in physics or quantum mechanics, such approach will not work here, because D. J. Pons, A. D. Pons, and A. J. Pons reject the standard physics and quantum mechanics in favour of the NLHV approach and design thinking ("taking the functional requirements [...] and inferring the requisite attributes" (Pons et al., 2013b, p. 25)). Therefore, more general arguments need to be employed to uncover the shortcomings of this theory.

\subsection{Arguments Against the Cordus Account of Cognitive Time}

I will leave, for now, the issue of the connectedness of time and fabric time, as discussing them later on, once we get to a more fundamental level, will yield more benefit. Presently, as stated above, I will start by discussing the cognitive time.

D. J. Pons, A. D. Pons, and A. J. Pons take time, as perceived by humans, to be a cognitive construct, build-up of chemical operations (the organic time level) of the organic components, and the arrow of time/irreversibility that appeared at the decoherent assembly level. However, human cognition, or perception of time is much more complex than what the above theory suggests. There are several things to consider.

\subsubsection{The Uniformity of the Flow of Time}

Firstly, this matter-emergent time theory offers no explanation as to why humans construct a uniform, flowing time. After all, the oscillation frequency of a decoherent system is neither uniform and nor synchronous with other decoherent assemblages that surround it. Its apparent phase-like nature suggests rather jumps from one unit of time to another than an even flow. The events humans perceive appear to flow seamlessly from one to another, with no discontinuous "jumps". The structure this theory offers provides no such seamlessness for our experience. Time seems to be discrete rather than continuous. The uniformity of the perceived flow of time is thus not accounted for.

\subsubsection{Specious Present and the NOW}

Next, one might ask about the NOW that the theory interprets as "a cognitive effect associated with consciousness, memory, and the process of thought" (Pons et al., 2013b, p. 34). Clearly, the issue of memory is much more complex than what this brief passage suggests. For years now philosophers have been wondering how our experience of the present can be explained (Note 6). There are at least two approaches to our experiencing the present: the extensional approach to specious present and the retentional approach to specious present, which are currently being analysed within the framework of various theories of time (Note 7). If we were to take the definition of NOW presented in D. J. Pons, A. D. Pons, and A. J. Pons (2013b) seriously, we would have to conclude that it is the retentional approach to specious present that is the correct one, as it entails the function of a short-term memory. However, when looked at closely, there is no conclusive argument that forces us to reject the extensional approach. Moreover, an extension here could be interpreted as overlapping re-energising oscillations at an organic level. Therefore, the Cordus inspired theory of time gives us no sufficient reason to choose one interpretation of specious present over the other.

Still, the problem runs deeper once we start concerning ourselves with the duration of what we call the present. What D. J. Pons, A. D. Pons, and A. J. Pons suggest is that there is one single NOW. They do not stipulate however the duration of this one single NOW. The durative problem of the present, in terms of cognition, is a complex one, spanning across several possibilities (Note 8). We have to consider that our perception of time, much like time itself in Cordus theory, is multi-layered. It spans from the scale of neurological events (what Gallagher (2011) calls elementary scale), to future intentions, or F-intentions (at a narrative scale). This time perception may be broadened further when we consider the existential horizon of a human being (Note 9) which may create additional issues. More to the point, there is a difference between time and the concept of time-a distinction which plays an important role in time perception. Nosal (2006) stresses the difference between the external, conceptual time and internal biological rhythms. (Note 10) Due to limitations of attention and short-term memory, human beings rely heavily on "time prosthetics - external reference frames like natural cycles, clocks, calendars, activity schedules, plans etc. Therefore, the more reliant we are on 'external time', the more we can dampen, or ignore the patterns and rhythms of time conditioned by our own aspirations, needs, life goals."(Nosal, 2006, p. 14) The already mentioned F-intentions, and the just discussed life goals and aspirations cause further problems for Cordus theory of time. The way humans perceive time allows for planning for the future. In terms of relevance of time to psychology, the most prominent field is the psychology of personality. Lewin (1935) treats personality as an organ responsible for controlling time and achieving a desired future. This line of argumentation leads us to the most important issue: the relationship of consciousness and time as 
perceived by a human being. One way to look at it is to take a reductionist, functionalist, or eliminativist view of mind and consciousness (Note 11) (one may suppose that physicists like D. J. Pons, A. D. Pons, and A. J. Pons would lean to such interpretations). If we take this approach we will be dangerously close to petitio principii. It will be so, because mind/consciousness would either be a property of matter, somehow creating cognitive time which in itself is a property of matter, or we will deny the existence of mind/consciousness altogether, thus having nothing to create the cognitive time. Another way to look at this is to outright admit some form of dualism. One way seems confusing, the other one ontologically costly.

\subsubsection{Anomalous Nature of Level 4}

How then can human time perception agree with the account of Cordus theory? According to the latter, time perception is a cognitive construct, build-up of "time" at an organic level. Neurobiological events are clearly representative of this "time" at an organic scale. Therefore, they do not/cannot be a part of human time perception. Human time perception has phenomenological qualities which time at an organic level simply does not have. Time perception is a result of neurological processes, a cause-and-effect relationship, rather than material for constructing this perception. To put it in classical, Aristotelian terms (Note 12), neurological events are an efficient, not a material cause of our time perceptions. D. J. Pons, A. D. Pons, and A. J. Pons do state that neurological events cause us to have time perception (Level 4). The problem lies elsewhere. Transitions from a more basic to a higher level, for levels 1 through 3 are all identical in nature. Level 2 (coherent/decoherent assembly) arises because particules (level 1) "stack up" and interact with one another forming an assembly. Level 3 (Note 13) (organic time) arises because assemblies (level 2) "stack up" and interact with one another. Level 4 however is different. It does not arise because organic assemblies stack up. For one, it would be an extraordinary coincidence that such different assemblies (as one person differs significantly from another) would in each instant result in creation of mind/consciousness. So, cognitive time is significantly different from other levels. Such anomaly, although not problematic on its own, together with other delineated issues becomes a cause for concern.

\subsubsection{One NOW, or Many NOWs?}

Another point to make is the fact that each person may have a different span of NOW. For example, a person A and a person B may perceive events $p$ and $q$ as present. Then, one of the events, let's say $p$ moves out of person's A present, while remaining within the scope of person's B present. In such a case, there is not one NOW. If NOW is a subjective cognitive creation, then each person would have her own NOW - in the above case, instead of a single NOW, we would have $\mathrm{NOW}_{\mathrm{A}}$ and $\mathrm{NOW}_{\mathrm{B}}$. The question then would be: how are these different NOWs synchronised? More to the point, if we ship a single person out to Andromeda, according to the Cordus theory of time, that person shares our NOW, because there is a single NOW. This seems highly unlikely for the following reasons. Firstly, there is no absolute simultaneity, as prescribed by the special relativity. Secondly, the person at Andromeda, by virtue of the Cordus theory, creates time perception and the NOW. Persons left on Earth also create the NOW. The two NOWs are clearly not a single NOW, they are rather (SR) NOW Andromeda and NOW $_{\text {Earth }}$. Effectively, we could ship all people, one by one, to different, distant parts of the universe, thus creating as many NOWs as we have people to spare.

The above arguments make the Cordus theory of time interpretation of temporal cognition doubtful. Human time perception needs more than just events at an organic or chemical level. The sheer fact of interaction with our surroundings compels us to broaden the perspective in which we analyse this cognitive phenomenon.

The next troubling issue is found at the fabric time and connectedness level.

\subsection{Arguments Against the Global Picture of Fabric and Connectedness}

The theory states that particules, or more precisely, their reactive ends release discrete forces in all three directions. Each frequency cycle results in discrete forces being sent down the hyff (meaning "field" in conventional physics). This transmission influences other particules in the accessible universe. Conversely, all other particules in the accessible universe emit their forces at their frequency cycles, influencing all others. There is no case where a particule could be measured in isolation. If not for any other reason than because an observer, or a measuring apparatus would need to be present to perform the measurement, and to do so she or it would need to be present in the particule's past light cone. Therefore, because everything influences everything else (Note 14), there is no "own", "original", "intrinsic", or what have you, frequency of a particule. We never have frequency $f$ of a particule. All we have is frequency of a particule at a particular state of the accessible universe $f_{\text {Un }}$.

The nature of the fabric that D. J. Pons, A. D. Pons, and A. J. Pons present, together with the nature of assembly, 
renders the account of individual oscillation frequency of a particule as fundamental time invalid. The necessary interconnectedness of particules prevents us from ever being able to determine individual oscillation frequency of a lone particule. If we accept the fabric as it is presented, than we will need to stop at an assembly level. This applies to both coherent and decoherent assemblies, because even if an assembly is coherent, it still interacts with the rest of the universe (Note 15).

There is one more troubling issue here. Granted, reactive ends of a particule may be spatially distant, yet still communicate with each other instantaneously/superluminally. However, two different particules do not propagate their forces (EMG) at superluminal speed. It is for this reason that we talk of light cones. A particule located at a particular point in space should only be influenced by particules (assemblies) located in its past light cone, and should only be able to influence particules in its future light cone. According to Cordus conjecture, reactive ends of a particule may well be at opposite edges of the universe and still communicate with one another instantaneously via fibril. What this means is that one particule could at most influence two future light cones and be influenced by two past light cones. Factoring into this equation the fact that a particule's behaviour depends on its surroundings (past light cone), would mean that the influence of a particular past light cone is reflected not only in the proximal future light cone, but also in the distant one (transmitted via a particule's fibril) This gives an impression that the entire universe is connected, but such impression would be unwise. For the entire universe to be connected, every particule would need to be spatially extended in such a way that its reactive ends were located in two different light cones. And although it is not logically impossible, such assumption would be blatantly unscientific, as it cannot be verified, or falsified.

One more thing should be added here, although it concerns more the decoherent assembly level, rather than fabric time. D. J. Pons, A. D. Pons, and A. J. Pons state that "the physical size of the body [is] the main scaling factor" (Pons et al., 2013b, p. 25). Surely though it is not the size, but the complexity of an assembly. A simple example should suffice: it does not really matter how large a body of superfluid we take, it still is a coherent assembly. Similarly, we should be able to easily find a decoherent two-particule assembly.

\subsection{Argument Against the Fundamental Level}

The key argument that renders this theory invalid lies with its central assumption-time is the oscillation frequency of a particule. There is no problem with particule as such, or with its internal structure. We may well assume that Cordus conjecture is correct and that such structure is acceptable. The main problem of this theory is the assumption delivered in section 3.3 of the paper. Authors state the following:

"it is a reasonable assumption that at least the ticks of time are really represented in the mechanism of the clock. Although that mechanism is uncertain, we accept that it involves transient atomic behaviour, and therefore the ticks of time are associated with atomic events." (Pons et al., 2013b)

And a little later:

"time arises as the frequency cycles of a NLHV particule, and consequently that time depends on how the particules are assembled into matter and hence physical bodies." (Pons et al., 2013b)

The question we need to ask first is: what does an atomic clock measure? An atomic clock is said to measure time and frequency by means of recording the "frequency of the microwave electromagnetic radiation emitted or absorbed by the quantum transition (energy change) of an atom or molecule [commonly caesium, or ammonia-K.Ł.]" (Note 16). So, basically, an atomic clock records the frequency of microwaves emitted by electrons when they change energy levels (Note 17). But how exactly (apart from the obvious-accuracy) is it different from a grandfather clock. We invented the grandfather clock just like we invented the atomic clock. We claim that both of these items measure time, but they really do not. Both of these items measure the change in energy level - the former with regard to the potential energy stored inside its spring, the latter with regards to the energy stored in an electron. What we do is, we transfer these "ticks" of a (any) clock onto what we conceive as time - the pace of change that surrounds us. In reality however no clock measures time per se.

Defining time as frequency of oscillation is dangerously close to petition principii reasoning. Frequency is in itself a temporal concept. Most common definitions of frequency are: (i) the rate at which something occurs over a particular period of time or in a given sample; and (ii) the rate per second of a vibration constituting a wave, either in a material (as in sound waves), or in an electromagnetic field (as in radio waves and light). Therefore, time cannot be defined as oscillation frequency of a particule, because frequency itself requires time.

A very similar argument runs for an atomic clock. We design an atomic clock to measure time, and then we equate what is being measured, with how it is being measured. The what in this case is time, and the how is the microwaves emitted by electrons. The method of measurement is not the same thing as what is being measured. 
Following this line of thought one might state that the scale on a measuring rod the length of 1 meter is in fact space. It clearly is not, it is just used to measures space.

\section{Conclusions}

Although the matter-emergent theory of time based on Cordus conjecture fails, it nevertheless provides an interesting direction in thinking about time. The multi-layered approach offers a plausible model for pulling together the different theories of time found in quantum mechanics, general relativity, psychology, philosophy and other. Perhaps a departure from particules by replacing them with a different concept will offer a more feasible theory of time.

\section{Acknowledgements}

I would like to thank Jerzy Gołosz and Dagna Skrzypińska, both of the Jagiellonian University, for reviewing an early draft of this paper.

\section{References}

Atomic Clock. (2013, November 18). In Wikipedia, the free encyclopedia. Retrieved November 19, 2013, from http://en.wikipedia.org/wiki/Atomic_clock

Block, N. (1980). What is Functionalism? Readings in Philosophy of Psychology (Vol. 1). Cambridge/MA: Harvard University Press.

Churchland, P. (2006). A Neurophilosophical Slant on Consciousness Research. Progress in Brain Research, 149, 285-293. http://dx.doi.org/10.1016/S0079-6123(05)49020-2

Crisp, T. M. (2004). Presentism. In M. J. Loux \& D. W. Zimmerman (Eds.), The Oxford Handbook of Metaphysics. Oxford: Oxford $\quad$ University http://dx.doi.org/10.1093/oxfordhb/9780199284221.003.0009

Dainton, B. (2011). Time, Passage, and Immediate Experience. In C. Callender (Ed.), The Oxford Handbook of Philosophy of Time. Oxford: Oxford University Press. http://dx.doi.org/10.1093/oxfordhb/9780199298204.003.0013

Encyclopaedia Britannica.

Falcon, A. (2012). Aristotle on Causality. In E. N. Zalta (Ed.), The Stanford Encyclopedia of Philosophy. Retrieved from http://plato.stanford.edu/archives/win2012/entries/aristotle-causality/

Feigl, H. (1958). The "Mental" and the "Physical". In H. Feigl, M. Scriven \& G. Maxwell (Eds.), Concepts, Theories and Mind-Body Problem (pp. 370-497). Minneapolis: University of Minneapolis Press.

Fine, K. (2004). The Problem of Possibilia. In M. J. Loux \& D. W. Zimmerman (Eds.), The Oxford Handbook of Metaphysics. Oxford: Oxford University http://dx.doi.org/10.1093/oxfordhb/9780199284221.003.0007

Gallagher, S. (2011). Time in Action. In C. Callender (Ed.), The Oxford Handbook of Philosophy of Time. Oxford: Oxford University Press. http://dx.doi.org/10.1093/oxfordhb/9780199298204.003.0014

Leggett, A. J. (2003). Nonlocal Hidden-Variable Theories and Quantum Mechanics: An Incompatibility Theorem. Foundations of Physics, 33(10), 1473-1474. http://dx.doi.org/10.1023/a:1026096313729

Lewin, K. (1935). A Dynamic Theory of Personality. New York: McGraw-Hill.

Lewis, D. (1986). On the Plurality of Worlds. Oxford: Blackwell.

Lyre, H. (2008). Time in philosophy of physics: The central issues. Physics and Philosophy, 1-23. Retrieved from http://hdl.handle.net/2003/25146

Markosian, N. (2013). Time. In E. N. Zalta (Ed.), The Stanford Encyclopedia of Philosophy. Retrieved from http://plato.stanford.edu/archives/fall2013/entries/time/

Nosal, C., \& Bajcar, B. (2004). Czas Psychologiczny: Wymiary, Strukture, Konsekwencje. Warszawa: Instytut Psychologii PAN. [Psychological Time: Dimensions, Structure, Consequences]

Nosal, C. (2006). Czas w umyśle: Psychologiczne modele temporalności. In W. Dziarnowska \& A. Klawiter (Eds.), Mózg i jego umyst. Poznań, Zysk i S-ka. [Time in Mind: Psychological Models of Temporality. In: The Brain and its Mind]

Pons, D. J., Pons, A. D., \& Pons, A. J. (2011). Gravitation, Mass and Time. (Cordus in extremis Part 4.3) viXra, 1104.0029, 1-14. Retrieved from http://vixra.org/pdf/1104.0029v1.pdf 
Pons, D. J., Pons, A. D., \& Pons, A. J. (2012). Wave-particle duality: A conceptual solution from the cordus conjecture. Physics Essays, 25(1), 132-140. Retrieved from http://physicsessays.org/doi/abs/10.4006/0836-1398-25.1.132

Pons, D. J., Pons, A. D., \& Pons, A. J. (2013a). Synchronous interlocking of discrete forces: Strong force reconceptualised in a NLHV solution. Applied Physics Research, 5(5), 107-126, http://dx.doi.org/10.5539/apr.v5n5p107

Pons, D. J., Pons, A. D., \& Pons, A. J. (2013b). Time: An emergent property of matter. Applied Physics Research, 5(6), 23-47, http://dx.doi.org/10.5539/apr.v5n6p23

Putnam, H. (1975). Philosophy of Our Mental Life. In: H. Putnam, Mind, Language and Reality. Philosophical Papers (Vol. 2, pp. 291-303). Cambridge/MA: Harvard University Press. http://dx.doi.org/10.1017/CBO9780511625251.016

Rea, M. (2004). Four-Dimensionalism. In M. J. Loux \& D. W. Zimmerman (Eds.), The Oxford Handbook of Metaphysics. Oxford: Oxford University http://dx.doi.org/10.1093/oxfordhb/9780199284221.003.0010

Sider, T. (2004). Reductive Theories of Modality. In M. J. Loux \& D. W. Zimmerman (Eds.), The Oxford Handbook of Metaphysics. Oxford: Oxford University Press. http://dx.doi.org/10.1093/oxfordhb/9780199284221.003.0008

\section{Notes}

Note 1. Other important issues analysed by Pons et al. in light of the Cordus conjecture are strong force (Pons, D. J., Pons, A. D. \& Pons, A. J. 2013a), waive-particle duality and quantum entanglement (Pons, D. J., Pons, A. D. \& Pons, A. J., 2012).

Note 2. D. J. Pons, A. D. Pons, and A. J. Pons define decoherence in the following way "The Cordus theory anticipates three mechanisms for decoherence. First, a coherent material cannot accept internal shear velocity. Second, higher temperatures lead to decoherence because phonons (internal thermal vibrations) disturb the stability. Third, more complex assemblies of matter are harder to put into coherence, and the complicating factors are the number of components in the assembly, and the variety of species (simplicity and purity)" (Pons et al., 2013b, p. 43).

Note 3. D. J. Pons, A. D. Pons, and A. J. Pons claim that this is also where entropy emerges. Although I find myself doubtful as to the validity of this statement regarding entropy (at least as viewed by Shannon), the scope of this paper is much too narrow to thoroughly investigate this issue.

Note 4. See Pons et al., 2013b, p. 32, and p. 46, for detailed examples of time dilation and gravitational time dilation respectively.

Note 5. For an account of possible worlds theory see Fine (2004), Sider (2004), also, for an original account Lewis (1986).

Note 6. For an account of human experience of time, and both extensional and retentional specious present, see Dainton (2011).

Note 7. Most notably the theories concerned are block theory, growing block theory, growing block and glowing edge theory, moving spotlight theory, and presentism. For an account of these theories see Crisp (2004), Markosian (2013) and Rea (2004). For extended and retentional models of specious present analysis see Dainton (2011).

Note 8. For a good account of time perception and duration of the present see Gallagher (2011).

Note 9. Nosal openly states that "it is not clear whether basic neurological processes conditioning the temporality also exert influence on superordinate levels (cognitive, existential)". p. 13. Own translation.

Note 10. For a complete account of psychological approach to time see Nosal, and Bajcar (2004).

Note 11. For views on eliminativism see Churchland (2006); for an account of functionalism see Putnam (1975), or Block (1980); Feigl (1958) offers an excellent delineation of reductionism.

Note 12. For an account of Aristotelian four causes see Falcon, A. (2012) "Aristotle on Causality". The Stanford Encyclopedia of Philosophy, ed. Edward N. Zalta.

Note 13. An important thing to mention is the fact that organic assemblies differ significantly among themselves. 
Cells of each person (or organism) age at different rates. Also, the neurotransmitters are produced at different rates and magnitudes. As such, rhythms do differ making perception of a uniform time tricky at best.

Note 14. In the accessible universe.

Note 15. A similar notion was introduced by Mach for measuring ephemeris time.

Note 16. Entry from Encyclopaedia Britannica.

Note 17. For a brief, but accurate description of an atomic clock see Wikipedia entry "Atomic clock".

\section{Copyrights}

Copyright for this article is retained by the author(s), with first publication rights granted to the journal.

This is an open-access article distributed under the terms and conditions of the Creative Commons Attribution license (http://creativecommons.org/licenses/by/3.0/). 